\title{
Criminologie
}

\section{La justice des mineurs au Québec : 25 ans de transformations (1960-1985)}

\section{Jean Trépanier}

Volume 19, numéro 1, 1986

Politiques et pratiques pénales. 25 ans de réflexion et d'action

URI : https://id.erudit.org/iderudit/017232ar

DOI : https://doi.org/10.7202/017232ar

Aller au sommaire du numéro

Éditeur(s)

Les Presses de l'Université de Montréal

ISSN

0316-0041 (imprimé)

1492-1367 (numérique)

Découvrir la revue

Citer cet article

Trépanier, J. (1986). La justice des mineurs au Québec : 25 ans de transformations (1960-1985). Criminologie, 19(1), 189-213.

https://doi.org/10.7202/017232ar
Résumé de l'article

This paper provides an overview of some of the changes undergone by the juvenile justice system in Quebec since 1960, with a particular attention to the evolution of its legal framework. Major legislative changes have stressed children's rights and diversion. The underlying philosophy of the law has been changed extensively, particularly concerning young offenders. Statistics reveal that the number of court referrals has increased considerably over time, and that diversion policies have been unable to change this trend. Juvenile court dispositions seem to show a greater degree of intervention than before. 


\section{LA IUSTICE DES MINEURS AU QUÉBEC : 25 ANS DE TRANSFORMATIONS (1960-1985)}

Jean Trépanier*

This paper provides an overview of some of the changes undergone by the juvenile justice system in Quebec since 1960 , with a particular attention to the evolution of its legal framework. Major legislative changes have stressed children's rights and diversion. The underlying philosophy of the law has been changed extensively, particularly concerning young offenders. Statistics reveal that the number of court referrals has increased considerably over time, and that diversion policies have been unable to change this trend. Juvenile court dispositions seem to show a greater degree of intervention than before.

\section{INTRODUCTION}

Tribunal de la jeunesse, directeur de la protection de la jeunesse, substitut du procureur général, délégué à la jeunesse, centre d'accueil, famille d'accueil, aide juridique, Comité de la protection de la jeunesse, centre de services sociaux... On pourrait ainsi dresser une longue liste de termes qui font aujourd'hui partie du vocabulaire quotidien des intervenants dans la justice des mineurs québécoise, mais qui, il y a vingt-cinq ans, auraient fait dresser l'oreille et peut-être suscité la question : «Pardon? De quel pays êtes-vous, je vous prie?» Car au Québec, on parlait alors de la Cour de bien-être social, de son contentieux, d'institutions, des foyers nourriciers, des agences familiales, et ainsi de suite. Question de vocabulaire? Dans une certaine mesure, sans doute : la réalité ne change pas du seul fait qu'on la nomme différemment. Mais il y a plus, beaucoup plus. Un intervenant de 1960 qu'on plongerait sans transition dans la justice des mineurs de 1985 serait estomaqué. La Cour de bien-être social n'était pas à l'époque encore implantée dans tous les districts judiciaires, de telle façon que certaines causes de mineurs étaient encore entendues par la Cour de magistrat. Elle ne comptait alors pour l'ensemble du Québec qu'une dizaine de juges. Le nombre de juges autorisés par la Loi

- Professeur agrégé à l'École de criminologie de l'Université de Montréal. 
sur les tribunaux judiciaires ${ }^{1}$ étant aujourd'hui de 43 . Les avocats de la défense n'y faisaient que de fort rares apparitions, au cours desquelles ils se sentaient d'ailleurs plongés dans un univers n'ayant rien en commun avec les autres tribunaux. Les juges, siégeant souvent dans des locaux exigus et parfois vétustes, y faisaient figure de bon père de famille. Le climat général était à l'absence de formalités. Les causes étaient entendues et les décisions prises beaucoup plus rapidement qu'aujourd'hui. Les échanges entre juge et agent de probation (déterminants pour le choix de la sanction) se tenaient régulièrement hors de la présence du jeune et de ses parents, que l'on informait ensuite de la décision sans nécessairement les consulter (le style personnel du juge y était pour beaucoup). Le fait que les décisions étaient officiellement prises en fonction de l'intérêt du jeune tenait lieu de droits à ce dernier et à ses parents. Les policiers exerçaient leur discrétion de référer les affaires au tribunal ou de ne pas le faire, mais le terme (pas très heureux) de "déjudiciarisation» n'avait pas encore été inventé par la Commission de réforme du droit du Canada ${ }^{2}$. Directeurs de la protection de la jeunesse et centres de services sociaux n'avaient pas encore vu le jour; assistés des agents de probation, les juges avaient un contact direct avec les ressources. Sauf quelques exceptions, les intervenants étaient aussi animés par l'enthousiasme d'aider les jeunes qu'ils étaient peu formés.

Ces quelques observations suffisent à elles-seules pour illustrer comment des changements majeurs sont survenus au cours de ce quart de siècle. Le cadre forcément restreint du présent article ne permettant pas de les évoquer tous, nous en aborderons quelques aspects en jetant un bref coup d'œil sur les régimes visant respectivement la protection de l'enfance en danger et la réaction sociale à la délinquance juvénile, concentrant spécialement notre attention sur la réforme du cadre légal qui a marqué cette période de façon particulière.

\section{LA PROTECTION DE L'ENFANCE EN DANGER}

Les premières lois québécoises visant à protéger les mineurs en danger remontent au siècle dernier. Une première ${ } \mathrm{i}^{3}$, datant de

1. L.R.Q., c. T-16, a. 110.

2. Canada, Commission de Réforme du Droit (1975), la Déjudiciarisation, Ottawa, Information Canada.

3. Loi des écoles d'industries, S.Q., 1869, c. 17. 
1869, permettait à un magistrat de placer dans une école dite d'industrie des enfants errants, sans moyen d'existence ou fréquentant la compagnie de voleurs de profession, de même que les enfants que leurs parents ne pouvaient contrôler et ceux qui, soutenus par une institution de charité, étaient jugés réfractaires ou avaient un parent qui avait été trouvé coupable d'une infraction passible d'emprisonnement. Par la suite, cette responsabilité fut transférée des mains des magistrats vers les tribunaux de la jeunesse. La Loi des écoles d'industries fut amendée à diverses reprises pour devenir ensuite Loi des écoles de protection de la jeunesse $e^{4}$, puis, il y a vingt-cinq ans, Loi de la protection de la ieunesse $e^{5}$.

La définition de ce qu'était un enfant en danger avait alors évolué vers la notion d'enfant "particulièrement exposé à des dangers moraux ou physiques, en raison de son milieu ou d'autres circonstances spéciales» ${ }^{6}$. Une constante était cependant demeurée à travers ces changements : la porte d'entrée du réseau de la protection de la jeunesse était située au tribunal. Lorsqu'on estimait qu'un enfant était en danger, c'est au tribunal qu'on le référait. Le juge devait alors dans chaque cas tenir une enquête afin de déterminer si l'enfant était bien en danger, pour ensuite émettre une ordonnance qui pouvait rendre accessible les mesures de protection.

La réforme des services sociaux étant survenue au début des années 1970, il restait à son maître, le ministre des Affaires sociales de l'époque, Monsieur Claude Castonguay, à compléter son projet en procédant à la révision de la Loi de la protection de la jeunesse. Il tenta de le faire en 1972. En situant ailleurs qu'au tribunal la porte d'entrée du réseau de la protection de la jeunesse, son projet de ${ }{ }^{i^{7}}$ reflétait le consensus qui avait déjà commencé à se faire à l'effet que le processus judiciaire apparaissait inutile et encombrant pour de nombreux cas où les parties en cause s'entendaient sur la nature du problème et des mesures à prendre. Le projet de loi rencontra toutefois une opposition qui amena le gouvernement à

4. Loi des écoles de protection de la jeunesse, S.Q., 1950, c. 11.

5. Loi de la protection de la jeunesse, S.Q., 1959-60, c. 42.

6. Loi de la protection de la jeunesse, a. 15.

7. Projet de loi 65 sur la protection de la jeunesse, 1972, Assemblée nationale du Québec, 3e session. 29e législature. 
ne pas forcer son adoption. Un amendement ${ }^{8}$ fut cependant apporté sans bruit à la loi en 1974, à l'instigation du ministre de la Justice, pour permettre de régler sans avoir recours au processus judiciaire, une catégorie particulière d'affaires de protection : les cas d'enfants victimes de mauvais traitements physiques. Après une nouvelle tentative infructueuse en 1975, la loi fut finalement révisée en $1977^{9}$. La nouvelle loi devait entrer en vigueur en janvier 1979.

La déjudiciarisation de la protection de la jeunesse constitue sans doute l'un des deux traits marquants de cette législation. C'est désormais le centre de services sociaux et non le tribunal qui contrôle l'entrée du système. C'est au directeur de la protection de la jeunesse que l'on signale tout cas d'enfant dont la sécurité ou le développement semble compromis. C'est à lui que revient de décider, après évaluation, si le recours à des mesures de protection pourra se faire sur une base volontaire ou si une intervention du tribunal sera demandée. C'est sur lui enfin que repose la responsabilité de l'application des mesures, qu'elles soient ou non volontaires.

Le bilan qui est fait de cet aspect de la loi est généralement présenté comme positif ${ }^{10}$ : on est satisfait de pouvoir régler d'un commun accord et dans un cadre souple et informel des affaires ne requérant pas vraiment d'intervention judiciaire. Les rôles respectifs du social et du judiciaire semblent clarifiés : le social est responsable des décisions volontaires et de l'application des mesures, alors que le tribunal arbitre et ordonne là où une intervention d'autorité est requise. Cela n'empêche pas certaines interrogations de subsister. Dans quelle mesure les ententes que l'on dit volontaires peuvent-elles être vraiment volontaires pour des clients qui savent qu'on les référera vraisemblablement au tribunal s'ils refusent leur accord? Par ailleurs, compte tenu des importantes conséquences à long terme que comporte souvent le retrait d'un jeune de son milieu familial, on peut se demander s'il ne serait pas préférable d'assujettir un tel placement (particulièrement en centre d'accueil) à la décision du tribunal? Le débat

8. Loi concernant la protection des enfants soumis à des mauvais traitements, L.Q. 1974, c. 59.

9. Loi sur la protection de la jeunesse, L.R.Q., c. P-34.1.

10. Voir par exemple QUÉBEC (1982), Rapport de la commission parlementaire spéciale sur la protection de la jeunesse, p. 310. Québec: Assemblée nationale du Québec (Rapport Charbonneau). 
judiciaire n'est-il pas de nature à prévenir que des décisions de placement ne soient prises trop hâtivement (ce qui revêt une importance toute spéciale dans les cas où les parents eux-mêmes souhaitent que le placement ait lieu)? En fait, ces interrogations sont proches parentes de cette seconde préoccupation qui a marqué la réforme de 1977 : la protection des droits des enfants.

La raison d'être des institutions de protection de la jeunesse est de répondre aux besoins de jeunes qui sont en danger. Ces organismes existent et fournissent leurs services dans l'intérêt des enfants. Aussi, pendant longtemps n'a-t-on vu aucune pertinence à parler de droits des enfants. Ceux-ci avaient besoin de mesures protectrices; dès lors qu'on les leur offrait, tout allait bien. Sans doute y avait-il des déficiences dans la dispensation de ces services; mais celles-ci étaient définies en termes d'améliorations à apporter aux ressources plutôt qu'en termes de violations de droits des jeunes. Comme il sera mentionné plus loin, une remise en question s'amorça à cet égard il y a une vingtaine d'années à l'égard des mineurs délinquants : si bien intentionnées soient-elles, des interventions d'autorité touchant l'intimité et la liberté des mineurs et de leurs familles ne devaient-elles pas être exercées à l'intérieur de certaines balises qu'il serait interdit de dépasser? Cette réflexion fut assez rapidement étendue aux mineurs en danger : n'intervenait-on pas d'autorité dans leur vie à eux aussi? C'est ce qui amena le législateur québécois à reconnaître formellement un certain nombre de droits aux enfants en danger. Les protections par la procédure judiciaire furent tout d'abord renforcées : d'informelle qu'elle était auparavant, la procédure à suivre devant le tribunal pour établir l'état de danger et le besoin de mesures protectrices devint celle qui s'applique normalement en matière civile. Mais le législateur ne s'arrêta pas là : il inséra au tout début de la loi un chapitre presque entièrement consacré aux droits des enfants ${ }^{11}$.

Ce chapitre spécial, après avoir situé certaines responsabilités des parents et des intervenants, affirme le principe suivant lequel les décisions prises à l'endroit des mineurs en danger doivent l'être dans leur intérêt et dans le respect de leurs droits. La loi fait obligation aux divers intervenants d'informer l'enfant et ses parents de leurs droits, et notamment de celui de consulter un avocat. Dans

11. Loi sur la protection de la jeunesse, a.3-11.3. 
leurs décisions, ces mêmes personnes doivent tendre à maintenir l'enfant dans sa famille; si cela est impossible, il faut offrir à celui-ci soins et conditions de vie se rapprochant le plus possible d'un milieu familial normal. Avant qu'une décision ne soit prise (que ce soit par le juge ou un intervenant social), on doit donner à l'enfant et à ses parents l'occasion de se faire entendre. Un certain nombre d'autres droits sont précisés, notamment à l'intention des mineurs faisant l'objet d'un placement.

Dans l'ensemble, il existe un consensus à l'effet que, en matière de protection de l'enfance en danger, cette réforme apportée par la loi de 1977 constitue un net progrès sur la situation antérieure. Ce n'est d'ailleurs pas sans raison que, réévaluant la Loi sur la protection de la jeunesse, une Commission parlementaire spéciale présenta en 1982 des recommandations ${ }^{12}$ qui ne touchaient en rien aux fondements de l'approche adoptée en 1977. Aussi les amendements apportés à la loi en 1984 se limitèrent à régler des problèmes ponctuels. Nous ne possédons pas de données chiffrées nous permettant d'avoir une vue d'ensemble systématique de l'évolution du recours aux mesures de protection au cours des 25 dernières années. Nous devons nous en remettre au sentiment qui semble prédominer à l'effet que, un quart de siècle après qu'une première loi ait officiellement porté le titre de Loi de la protection de la jeunesse, c'est largement avec satisfaction que semble perçue l'évolution suivie.

La même conclusion émerge-t-elle de la rétrospective que l'on peut faire en matière de réaction sociale à la délinquance juvénile?

\section{LA RÉACTION SOCIALE À LA DÉLINQUANCE}

Tout comme celle qui régit la protection de l'enfance en danger, la législation applicable aux mineurs délinquants a connu au cours da la même période des remises en question et des changements majeurs. Voyons-en les grandes lignes, après quoi nous tirerons quelques observations de données relatives à l'évolution de la clientèle et des décisions de la justice des mineurs.

\section{A. LA LÉGISLATION}

$\mathrm{Si}$ les premières dispositions législatives canadiennes relatives aux mineurs délinquants remontent à 1857, c'est en 1908 que le

12. Québec (1982), cf. sup., note 10. 
Parlement fédéral adopta la loi $^{13}$ qui devait graduellement permettre le développement des "cours des jeunes délinquants». Au Québec comme dans les autres provinces, ces tribunaux spécialisés ne furent implantés qu'avec le temps. Certains districts n'en étaient même pas encore pourvus au moment où, en 1961, la nomination du Comité MacLeod enclencha à Ottawa le processus de révision de la Loi sur les jeunes délinquants. Publié en 1965, le rapport ${ }^{14}$ de ce comité fut suivi en 1967 d'un avant projet de loi $^{15}$ puis, en 1970 , d'un projet de loi $^{16}$. Confronté à une opposition imprévue, le gouvernement ne força pas l'adoption du projet de loi et nomma un nouveau comité qui fit rapport en $1975^{17}$. Pour éviter un nouvel échec politique, le gouvernement fit procéder à une consultation nationale sur ce rapport avant de rendre public, en 1977 , les grandes lignes d'un avant-projet de $10^{18}{ }^{18}$. Deux ans plus tard, un changement de gouvernement donna lieu à de nouvelles déclarations d'intentions ${ }^{19}$. Enfin, après de nouvelles élections, un projet de loi fut déposé au Parlement en 1981, adopté en 1982 et mis en vigueur en $1984^{20}$. Presque un quart de siècle s'était écoulé c. J-3.

13. Loi concernant les jeunes délinquants, S.C., 1908, c. 40; S.R.C. 1970.

14. Canada, ministère de la Justice (1965), Délinquance juvénile au Canada : Rapport du Comité du ministère de la lustice sur la délinquance juvénile, Ottawa, Imprimeur de la reine (Rapport Macleod).

15. Canada, ministère du Solliciteur général (1967), Avant-projet aux fins d'étude: Loi concernant les entants et les adolescents, Ottawa, ministère du Solliciteur général.

16. Canada, Chambre des communes, Bill C-192 concernant les jeunes délinquants et abrogeant l'ancienne Loi sur les jeunes délinquants, 1re lecture le 16 novembre 1970.

17. Canada, Solliciteur général (1975), Loi sur les jeunes qui ont des démêlés avec la justice : Rapport du Comité du ministère du Solliciteur général sur les propositions formulées en remplacement de la Loi sur les jeunes délinquants, Ottawa, ministère do Solliciteur général (Rapport Tassé).

18. Canada, Solliciteur général (1977), Points saillants de l'avant-projes de loi sur les jeunes contrevenants, Ottawa, ministère du Solliciteur général.

19. Canada, Solliciteur général (1979), Propositions relatives à l'adoption d'une loi pour remplacer la Loi sur les jeunes délinquants. Ottawa, ministère du Solliciteur général.

20. Loi sur les jeunes contrevenants, S.C. 1980-82, c. 10. Cette nouvelle loi entra en vigueur le 2 avril 1984 (sauf en ce qui concerne les dispositions relatives à l'uniformisation à 18 ans de l'âge de la majorité pénale au Canada, qui n'entrèrent en vigueur qu'en avril 1985). 
depuis le début de l'entreprise de révision, en 1961. L'essentiel des travaux s'était fait à Ottawa, vu la compétence législative fédérale en la matière. Des commissions québécoises avaient toutefois abordé divers aspects des mêmes questions : il suffit de penser aux rapports Prévost ${ }^{21}$, Batshaw ${ }^{22}$ et Charbonneau ${ }^{23}$.

S'étalant sur plus de deux décades, ces divers documents témoignent clairement de l'évolution qui s'est faite au cours de cette période dans la conception que l'on se faisait de l'intervention de la justice des mineurs. Faute d'espace, limitons-nous à en observer les points saillants par rapport à trois thèmes : l'esprit de la loi, les droits des jeunes et la déjudiciarisation.

1. L'esprit de la loi

Avec la Loi sur les jeunes délinquants, un mineur trouvé coupable d'un délit devait être traité «non comme un contrevenant, mais comme quelqu'un qui est dans une ambiance de délit et qui, par conséquent, a besoin d'aide et de direction et d'une bonne surveillance ${ }^{24}$. Cette loi visait à ce que «chaque jeune délinquant soit traité, non comme un criminel, mais comme un enfant mal dirigé, ayant besoin d'aide, d'encouragement et de secours ${ }^{25}$. Le juge devait rendre des décisions en fonction du «bien de l'enfant et (du) meilleur intérêt de la société» ${ }^{26}$, sans que l'on ne précise si le second devait avoir préséance sur le premier en cas de conflit. Conçue d'abord en fonction de l'aide à apporter au mineur, cette loi ne faisait aucun appel à la responsabilité du jeune pour ses actes, ne faisait aucune référence à la proportionnalité de la mesure par rapport à la gravité de l'infraction et permettait le recours à des mesures à durée indéterminée jusqu'à ce que le jeune ait atteint l'âge de 21 ans. Dans un contexte où l'on voulait aider le jeune, pourquoi se serait-on imposé des limites dont l'effet aurait été

21. Québec, Commission d'enquête sur l'administration de la justice en matière criminelle et pénale au Québec (1970), la Sociêté face au crime, vol. 4, t. 1, la Cour de bien-être social, Québec, Éditeur officiel du Québec (Rapport Prévost).

22. Québec (1975), Rapport du comité d'étude sur la réadaptation des enfants et adolescents placés en centre d'accueil, Québec, ministère des Affaires sociales (Rapport Batshaw).

23. Voir sup., note 10.

24. Loi sur les jeunes délinquants, S.R.C. 1970, c. J.3.

25. Loi sur les jeunes délinquants, a. 38.

26. Loi sur les jeunes délinquants, a. 20(5). 
d'empêcher qu'on ne fournisse au mineur des services vus comme étant dans son intérêt?

Cette perspective faisait encore largement consensus il y a 25 ans. Aussi ne faut-il pas se surprendre que, tout en recommandant l'introduction de quelques balises, le Comité Macleod ait endossé l'orientation générale de la Loi sur les jeunes délinquants :

L'esprit fondamental de la loi n'est pas l'élément qui pose un problème; le problème réside plutôt dans le fait que la société n'a pas fourni au tribunal pour mineurs les ressources suffisantes pour réaliser l'esprit de la loi $^{27}$.

De la même façon, l'avant-projet de loi de 1967 et le projet de loi de 1970 établissent des limites au droit d'intervenir dans la vie des jeunes (notamment en limitant la durée des mesures à deux ou trois ans, selon le cas), mais conservent explicitement des principes axés sur l'aide ${ }^{28}$.

Ces principes étaient cependant de plus en plus remis en question. L'évolution des idées se traduisit, en 1975, dans le Rapport sur les jeunes qui ont des démêlés avec la justice, qui proposa que les jeunes soient tenus d'assumer la responsabilité de leurs actes. Ils ne devraient cependant pas l'être au même titre que les adultes, leur état leur créant "plus particulièrement (un) besoin d'aide, d'encouragement, de directives, et, le cas échéant, de surveillance, de discipline et d'autorité» ${ }^{29}$. Tout en étant tenus responsables de leurs actes, les jeunes devraient donc faire l'objet premièrement de mesures d'aide et, s'il y avait lieu, de mesures de surveillance. L'accent mis respectivement sur ces deux derniers pôles allait toutefois être inversé dans l'avant-projet de loi de $1977^{30}$ et, plus clairement encore, dans la Loi sur les jeunes contrevenants. De telle façon que, en plus d'affirmer la responsabilité des jeunes à l'endroit de leurs actes, la loi préconise aujourd'hui des mesures de "surveillance, discipline et encadrement», reléguant «conseils et assistance» au rôle de tempérament ${ }^{31}$. C'est la protection de la société qui désormais doit motiver les

27. Rapport Macleod (voir sup., note 11), p. 116-117.

28. Avant projet de loi sur les enfants et les adolescents (voir sup., note 12), a. 4 et 5 . Projet de loi C-192 (voir sup., note 13), a. 4.

29. Rapport Tassé (voir sup., note 14), p. 84.

30. Points saillants... (voir sup., note 15), p. 3 et 12.

31. Loi sur les jeunes contrevenants, a. 3(1) e). 
entraves à la liberté d'un jeune ${ }^{32}$; le bien du jeune n'est plus considéré comme un des grands objectifs de la loi. Les mesures imposées doivent être d'une durée déterminée à l'avance, dont le maximum tient compte de la gravité et du nombre des infractions. Auparavant largement positiviste, la législation comporte maintenant des éléments de nature nettement plus classique.

Est-ce à dire qu'une justice des mineurs, conçue au départ pour aider, est devenue essentiellement punitive? En fait, la réalité est plus complexe que les préceptes législatifs. Et il est vraisemblablement aussi faux d'affirmer de nos tribunaux actuels qu'ils ont oublié l'aide que de dire de nos juges d'il y a vingt-cinq ans qu'ils n'avaient pas recours à la punition comme sanction. Évoquant «les équivoques de la philanthropie», la Commission Prévost notait en 1970 que «le tribunal pour enfants souffre d'une opposition marquée entre les objectifs qu'on lui assigne et la pratique à laquelle il lui faut se restreindre et se résigner ${ }^{33}$. Ce sont pourtant ces nobles idéaux d'aide qui, même s'ils n'étaient pas atteints, étaient à l'origine de l'idée que les droits des jeunes n'avaient pas à être protégés au même titre que ceux des adultes.

\section{Les droits des jeunes}

La protection de l'individu contre une intervention abusive de l'État dans son intimité et sa liberté est au fondement même du droit pénal. Celui-ci contient tout un ensemble de règles dont l'objet premier est la protection des droits des citoyens. Cette conception est fondamentale en droit pénal classique, où l'État intervient en punissant l'individu, ce qui le place en quelque sorte dans une position d'agresseur. Dans ce contexte, l'État doit établir la légitimité de son intervention sur des assises solides, que l'on trouve dans des règles reconnaissant à tout citoyen accusé des droits lui permettant de se prémunir contre l'éventualité d'une punition injuste.

Comme il a été vu plus haut, l'optique des auteurs de la Loi sur les jeunes délinquants de 1908 se démarquait très nettement de la perspective classique qui inspirait (et inspire encore) le droit pénal canadien. Plutôt que punir le jeune, on disait vouloir l'aider. On n'était animé d'aucune intention malveillante à son endroit.

32. Loi sur les jeunes contrevenants, a. 3(1) f).

33. Rapport de la Commission Prévost (voir sup., note 18), p. 16. 
On voulait intervenir pour lui, non contre lui. Dès lors, pourquoi lui reconnaître les droits qui seraient en réalité pour lui un moyen de se protéger contre une aide bienveillante qu'on veut lui apporter? On adopta donc une loi qui ne reconnaissait pas aux mineurs les mêmes droits qu'aux adultes. À titre d'exemples, on peut surtout citer le recours à des procédures sommaires et moins formelles ou les restrictions imposées au droit d'appel. On peut aussi rappeler le fait que, quelle que soit l'infraction en cause, le jeune pouvait en principe faire l'objet de mesures ordonnées par le tribunal tant qu'il n'avait pas atteint l'âge de 21 ans, ce qui pouvait permettre des sanctions souvent beaucoup plus graves que celles dont un adulte aurait fait l'objet. Et ainsi de suite. L'accent était mis sur le bien de l'enfant, non sur ses droits.

Dans son rapport publié en 1965, le Comité MacLeod affirma son accord de principe avec la disposition de la Loi sur les jeunes délinquants prévoyant une procédure "aussi peu formaliste que le permet la bonne administration de la justice ${ }^{34}$; il recommandait en même temps que soient précisés et élargis quelques droits reconnus aux mineurs, notamment en matière d'appels. C'est toutefois des États-Unis que devaient venir, peu après, les véritables débats sur cette question. Dans quelques arrêts retentissant $\mathrm{s}^{35}$, la Cour suprême de ce pays mit en cause l'orientation de la justice des mineurs, concluant que :

... il y a lieu de craindre que l'enfant ne recevra que le pire de deux mondes : à savoir qu'il n'a ni les protections accordées aux adultes, ni le soin attentif et le traitement régénérateur réclamés pour les enfants ${ }^{36}$.

Si elle pouvait plus difficilement toucher au contenu des interventions, la Cour suprême américaine pouvait cependant exiger que l'on se préoccupe des droits des jeunes. Elle déclara inconstitutionnelles des législations qui privaient les mineurs délinquants de la protection du «due process of law». Bon nombre d'États durent alors rapidement revoir leurs lois afin de les rendre conformes aux exigences de la constitution en matière de protection des droits par la procédure. Ces changements furent entourés de débats importants qui trouvèrent des échos au Canada.

34. Rapport Macleod (voir sup., note 11), p. 152.

35. Les deux arrêts les plus connus à cet égard furent rendus dans les affaires Kent (1966, 383 U.S. 541) et Gault (1967, 387 U.S. 1).

36. Arrêt Kent (voir sup., note 32), p. 556. 
C'est dans ce contexte que, en 1970, la Commission Prếvost recommanda "que l'on étende aux jeunes contrevenants les précautions qui entourent l'arrestation, l'interrogatoire, la détention et le procès des prévenus adultes ${ }^{37}$. Sans aller aussi loin, le gouvernement fédéral proposa la même année, dans le projet de loi C-192, de mieux reconnaittre certains droits aux jeunes. Le comité formé suite à l'échec de ce projet de loi publia en 1975 un rapport ${ }^{38}$ où la protection des droits était affirmée de façon solennelle et imprégnait l'ensemble du projet. Le rapport se démarquait ainsi des documents qui l'avaient précédé et reflétait l'évolution rapide qui avait transformé les idées en dix ans sur cette question. Les avant-projets fédéraux de 1977 et 1979 allaient, comme la Loi sur les jeunes délinquants, reprendre dans l'ensemble la même orientation.

La loi maintenant en vigueur aujourd'hui affirme donc que les jeunes jouissent de droits et libertés assortis de garanties spéciales. Elle assure une meilleure protection des jeunes dans le cadre de l'interrogatoire policier. Elle définit mieux la procédure à suivre pour en arriver au choix d'une mesure. Si la procédure y est, pour les cas d'infractions sérieuses, plus sommaire que celle qui s'applique aux adultes, elle confère un droit d'appel comparable à celui dont jouissent ces derniers. Elle consacre le droit du jeune d'être informé de ses droits et assistés au cours des procédures qui le concernent. Elle affirme que les mesures prises à l'endroit du jeune ne doivent comporter qu'un minimum d'entrave à sa liberté. Des limites beaucoup mieux circonscrites sont fixées quant au droit de l'État d'intervenir dans la vie privée du jeune.

Ces nouvelles balises constituent autant de protection pour les droits des jeunes, particulièrement aux étapes policières et judiciaire du processus. Des interrogations subsistent toutefois concernant les protections qui peuvent être fournies à une autre étape, celle des mesures de rechange. On le verra plus bas, la Loi sur les jeunes contrevenants permet que, dans certains cas, des mesures de rechange volontaires soient substituées à l'intervention du tribunal. Le caractère volontaire de ces mesures constitue cependant un des points épineux de la déjudiciarisation. Quelque précaution qu'elle contienne, la loi ne peut changer le fait que le

37. Rapport Prévost (voir sup., note 18), recommandation 24, p. 116.

38. Rapport Tassé (voir sup., note 14). 
jeune doit choisir entre l'acceptation d'une mesure dont la nature lui est connue et un renvoi au tribunal dont l'issue est incertaine, et qu'il peut raisonnablement ne pas se croire dans une position où il pourrait négocier une solution plus acceptable s'il juge excessive la mesure proposée. Cette crainte doit être traitée d'autant plus sérieusement que la majorité des mineurs officiellement détectés comme délinquants proviennent de groupes à faible statut socioéconomique et, partant, sont parmi les plus démunis pour se défendre contre des interventions injustifiées dans leur vie. Si la présence occasionnelle d'avocats de la défense et la sensibilisation des intervenants à la nécessité des droits des jeunes peut réduire quelque peu l'ampleur du problème, celui-ci n'en demeure pas moins inhérent à la déjudiciarisation. L'insistance qu'on a placée sur les droits des jeunes aux étapes policière et judiciaire ne doit pas faire perdre de vue qu'on a introduit entre ces deux phases un autre stade où certains droits sont extrêmement difficiles à protéger.

Cette réserve mise à part, il faut conclure que la loi contient aujourd'hui une affirmation du droit des jeunes qui est le fruit d'un cheminement qui a marqué les deux dernières décades et qui eût été impensable il y a un quart de siècle. L'hypothèse d'un retour à une conception plus classique ne saurait expliquer que partiellement ce phénomène : à la différence des auteurs de la Loi sur les jeunes délinquants, le législateur contemporain a reconnu que, même lorsqu'on veut lui conférer une dimension bienveillante d'aide, une intervention non sollicitée peut donner lieu à des abus contre lesquels un mineur et sa famille doivent être protégés, au même titre qu'un accusé adulte a le droit d'être protégé contre une peine imméritée.

\section{La déjudiciarisation}

Séduits par les vertus des tribunaux pour mineurs dont ils voulaient assurer la mise en place, les sénateurs qui avaient fait adopter la Loi sur les jeunes délinquants en 1908 étaient peu portés à suggérer qu'il valait souvent mieux n'y avoir pas recours. Il faudra donc attendre un bon nombre d'années avant que ne surgisse le terme "déjudiciarisation». Non pas que la pratique en était inexistante : il est bien connu que les policiers n'ont toujours référé aux tribunaux qu'une partie des affaires concernant lesquelles ils avaient amassé les preuves nécessaires, se contentant de 
classer sans suite un nombre considérable d'affaires dont l'importance ne semblait pas justifier une intervention judiciaire. En ce qui concerne les mineurs délinquants, l'existence de ces pratiques a été établie par les travaux de Tardif et de Statistique Canada ${ }^{39}$. Quelque forme qu'elles aient pris, ces pratiques ont toutefois eu une caractéristique en commun : elles ont été informelles, non régies par des règles officielles. En 1965, le Rapport Macleod recommanda de formaliser les pratiques existantes en retirant aux policiers tout pouvoir discrétionnaire et en confiant à une personne nommée par le tribunal le soin de déterminer, à partir de critères préétablis, quels cas devraient faire l'objet de poursuites ${ }^{40}$.

Entre-temps, un phénomène nouveau était apparu aux ÉtatsUnis: l'émergence d'organismes (tels que les Youth Service Bureaus) auxquels des policiers référaient des mineurs plutôt que de les judiciariser. Une commission d'enquête présidentielle leur avait conféré leurs titres de noblesse ${ }^{41}$, deux ans après qu'en Grande Bretagne un livre blanc eût exprimé l'intention du gouvernement de formaliser le recours à des interventions autres que judiciaires ${ }^{42}$. S'appuyant notamment sur ces propositions et expériences étrangères, la Commission Prévost recommanda en 1970 que l'on suive cette même voie, le tribunal devant «apparaître en dernier ressort, seulement quand on a épuisé les possibilités de réorientation, de rééducation, d'entente avec les parents pour un traitement spécial... $\gg^{43}$ La même année, le projet de loi C-192 montrait que le gouvernement fédéral hésitait à s'engager dans de tels programmes sans supervision judiciaire: le projet de loi proposait qu'une affaire dont le tribunal était saisi puisse se régler sans audition moyennant l'accord du juge ${ }^{44}$.

39. Tardif, Guy (1976), le Contrôle de la délinquance: la police. Université de Montréal, École de criminologie, Rapport de recherche inédit. Statistique Canada (1977), aStatistiques préliminaires d'une enquête par échantillon sur les jeunes et la violation de la loi», Bulletin de service: la statistique policière, judiciaire et correctionnelle, 2, 1-9.

40. Rapport Macleod (voir sup., note 11), p. 121.

41. États-Unis, President's Commission on Law Enforcement and Administration of Justice (1967), Task Force Report : Iuvenile Delinquency and Youth Crime, p. 19. Washington, U.S. Government Printing Office (Rapport Katzenbach).

42. Grande Bretagne, Home Office (1965), The Child, the Family and the Young Offender, Londres, H.M.S.O. Cmnd. 2742.

43. Rapport Prévost (voir sup., note 18), p. 95.

44. Projet de loi C-192 (voir sup., note 13), a. 23. 
L'absence de cadre légal n'empêcha pas des expériences de voir le jour au cours des années suivantes. Ainsi, dès 1971, les avocats du contentieux de la Cour de bien-être social de Montréal se mirent à sélectionner les affaires déstinées au tribunal. Conçue au départ principalement pour les cas de jeunes résidant à l'extérieur de Montréal et pour lesquels un retour au foyer apparaissait plus approprié qu'une comparution devant le juge, cette procédure fut rapidement étendue à l'ensemble des cas de protection et de délinquance ${ }^{45}$. D'autres districts judiciaires adoptèrent la même façon de faire. Ainsi, lorsqu'en 1975 les gouvernements québécois ${ }^{46}$ et fédéral ${ }^{47}$ annoncèrent leur intention d'aller de l'avant en matière de déjudiciarisation de la délinquance, l'idée avait déjà largement fait son chemin dans le milieu des intervenants québécois. La formule proposée dans le rapport fédéral fut critiquée (particulièrement à l'extérieur du Québec), plus pour ses modalités que pour le fait qu'elle endossait le principe même de la déjudiciarisation. Aussi, le gouvernement fédéral annonca-t-il dès 1977 son intention de ne légiférer que sur le principe de la déjudiciarisation et sur quelques conditions minimales que devrait respecter toute intervention préjudiciaire, laissant aux provinces le soin de déterminer les modalités des programmes de déjudiciarisation ${ }^{48}$.

Mais le projet de loi fédéral que l'on attendait depuis 1976 ne venait toujours pas. Suivant en cela les intentions de celui qui l'avait précédé, le gouvernement en poste à Québec décida, moyennant quelques retouches, d'étendre aux affaires de délinquance le mécanisme de déjudiciarisation conçu pour les affaires de protection qui devait être proposé à l'Assemblée nationale dans le cadre de la révision de la Loi de la protection de la jeunesse. S'il allait soulever des problèmes d'ordre constitutionnel, ce choix était par ailleurs cohérent avec les vues que plusieurs défendaient à l'effet d'assimiler plus ou moins les affaires de délinquance aux affaires de protection. Et le processus retenu cadrait bien sur le

45. Pour un compte-rendu de cette expérience pour les années 19711976, voir Rosette Gagnon et Jean Trépanier (1985), la Déiudiciarisation à la Cour de bien-être social de Montréal entre 1971 et 1976, Université de Montréal, École de criminologie.

46. Québec, ministère des Affaires sociales (1975), Avant-projet de loi sur la protection de la jeunesse, Québec, ministère des Affaires sociales.

47. Rapport Tassé (voir sup., note 14).

48. Points saillants... (voir sup., note 15) , p. 6-7, 12, 14-16. 
fond avec les recommandations formulées en 1975 par le groupe de travail fédéral. L'Assemblée nationale du Québec adopta donc unanimement à la fin de 1977 la Loi sur la protection de la jeunesse qui, à compter de son entrée en vigueur au début de 1979, établissait à peu de choses près un mécanisme de déjudiciarisation commun aux affaires de délinquance et de protection. Désormais, les policiers comme les autres citoyens ne pourraient plus référer d'affaires directement au tribunal. Ils devraient signaler l'affaire au directeur de la protection de la jeunesse qui, après évaluation, déciderait de concert avec une personne désignée par le ministre de la Justice s'il y avait lieu de clore l'affaire, de proposer une mesure volontaire ou de référer le dossier au tribunal. On connaît la suite. La victime d'une infraction contesta avec succès la constitutionnalité de certains aspects de ce mécanisme. On entendit des rumeurs à l'effet que des corps policiers envisageaient d'en faire autant pour passer outre au mécanisme de déjudiciarisation et recouvrer l'accès direct aux tribunaux dont ils jouissaient avant 1979. Le tout s'ajoutant aux critiques que l'on entendait en divers milieux à l'égard de la nouvelle loi, le gouvernement québécois s'engagea à faire modifier celle-ci et, vers le même moment, l'Assemblée nationale chargea une commission spéciale de la réexaminer, tout particulièrement en ce qui concernait son application à la délinquance. La Commission Charbonneau recommanda de rétablir une nette distinction entre les affaires de délinquance et celles qui concernaient l'enfance en danger.

Entre-temps, le Parlement fédéral avait finalement adopté à l'été de 1982 la Loi sur les jeunes contrevenants qui, à compter d'avril 1984, remplacerait la Loi sur les jeunes délinquants. Régissant pour l'essentiel les interventions policières et surtout judiciaires, cette loi sanctionnait le principe de la déjudiciarisation et permettait le recours à des mesures de rechange dont les moda. lités seraient fixées par les provinces. Le gouvernement québécois fit adopter le 3 avril 1984 par l'Assemblée nationale une série d'amendements à la Loi sur la protection de la jeunesse, dont certains avaient pour effet de retirer de cette loi toute référence à la délinquance. Une série de décrets et d'arrêtés ministériels furent adoptés le 4 avril par le gouvernement pour préciser certaines modalités d'application de la loi fédérale que celle-ci laissait aux provinces en raison de leur juridiction en matière d'administration de la justice. Dès lors, on revenait à deux régimes distincts : l'un que traçait la Loi sur la protection de la jeunesse à l'intention des 
enfants en danger, et l'autre qu'établissait à l'endroit des mineurs délinquants la Loi sur les jeunes contrevenants complétée pour ses modalités d'application (dont le programme de mesures de rechange) par les décrets québécois. Fondée sur les interventions des substituts du procureur général et des directeurs de la protection de la jeunesse, une nouvelle procédure relativement complexe régissait désormais l'accès au tribunal et aux mesures de rechange en matière de délinquance.

Au terme d'une évolution commencée dans les années 1960, on avait sanctionné officiellement le recours à des mesures autres que l'intervention judiciaire et on l'avait encadré dans un régime distinct de celui régissant la protection de l'enfance en danger. Cette formalisation de la déjudiciarisation résolvait certains problèmes; elle en créait cependant peut-être de nouveaux, comme le suggère l'étude de l'évolution de la clientèle du Tribunal de la jeunesse.

\section{B. L'ÉVOLUTION DES CLIENTĖLES ET DES DÉCISIONS DU TRIBUNAL}

L'application de la Loi sur les jeunes délinquants ayant fait l'objet de rapports de Statistique Canada depuis plusieurs années déja $a^{49}$, nous disposons de quelques données permettant de situer quelques traits de l'évolution de la clientèle pénale des tribunaux pour mineurs au Québec et des décisions prises par les juges à son égard. Ces données ne sont toutefois pas sans problèmes. On peut d'abord entretenir à l'endroit de leur fiabilité les soupçons que l'on nourrit de façon plus générale à l'endroit des statistiques pénales, surtout lorsqu'on remonte dans le temps. Par ailleurs, deux problèmes spécifiques de comparabilité se posent dans le cas qui nous intéresse. Tout d'abord, jusqu'en 1967, les rapports de Statistique Canada concernant les jeunes délinquants ne faisaient état que des affaires concernant les mineurs de moins de 16 ans, s'adaptant ainsi au seuil d'âge de majorité pénale en vigueur dans le plus grand nombre de provinces canadiennes. Ce n'est que depuis 1968 que les données relatives au Québec incluent les

49. Statistique Canada (annuel), Jeunes délinquants, Ottawa, Statistique Canada. 
mineurs de 16 et 17 ans $^{50}$. Les données fournies plus bas doivent donc être analysées dans la perspective où ce dernier groupe d'âge en est exclu pour la période de 1960 à 1967. Par ailleurs, il faut noter que, de 1970 à 1973, un changement temporaire fit que les données publiées pour chaque province furent calculées sur la base du nombre de délinquants plutôt que sur celle du nombre d'affaires. Aussi, fut-il nécessaire de procéder à une estimation pour pouvoir fournir, pour ces quatre années, des données qui soient comparables à celles des autres années. Compte tenu des données disponibles, une estimation apparut possible ${ }^{51}$ pour éva. luer la clientèle du tribunal (tableau 1 et figure 1) mais elle sembla trop problématique pour que l'on en fît autant pour chiffrer le recours du tribunal aux diverses mesures (ce qui explique l'absence des années 1970-1973 du tableau 2 et de la figure 2). Enfin, les données les plus récentes datent de 1983; la période couverte ne concerne donc que l'application de la Loi sur les jeunes délinquants, la loj actuelle n'étant entrée en vigueur qu'en 1984. Ces réserves étant clarifiées, quelles observations peut-on tirer des données?

Les chiffres relatifs à l'évolution du nombre d'affaires référées au tribunal ${ }^{52}$ sont fournis en annexe dans le tableau 1 et illustrés dans le graphique 1. Quant aux données concernant les mesures prises par le tribunal, elles sont rapportées en annexe dans le

50. Les données relatives à ce groupe d'âge étaient, jusqu'en 1967. intégrées aux Statistiques de la criminalité. Elles n'étaient cependant pas rapportées d'une façon permettant de les isoler, de telle sorte qu'il n'a pas été possible de les incorporer dans les données fournies dans le présent article.

51. L'estimation a été faite comme suit pour les années 1970-1973. Statistique Canada rapportait dans une première section le nombre de délinquants, pour la Canada comme pour chaque province prise séparément, utilisant comme base l'infraction la plus sérieuse rapportée pour chaque délinquant au cours de l'année. Suivait dans une seconde section, une présentation pour le Canada (sans ventilation par province) du nombre d'infractions autre que la plus sérieuse rapportée pour chaque mineur. L'estimation de la portion québécoise du groupe d'infractions de la seconde section a été faite en concluant, pour chaque catégorie d'infractions apparaissant au tableau 1, quel pourcentage des cas de l'ensemble du Canada provenait du Québec dans la section $l$ du rapport, pour ensuite appliquer ce pourcentage à la section 2 du rapport et faire la somme des données fournies dans la section 1 et estimées pour la section 2 .

52. Le tribunal auquel il est fait référence ici est la Cour de bien-être social pour les années 1960-1978 et le Tribunal de la jeunesse pour les années subséquentes. 


\section{FIGURE 1}

Nombre d'alfaires référées au tribunal de la leunesse (Québec) pour des ${ }_{N} \quad$ infractions au Code criminel et autres lois fédérales, 1960-1983.

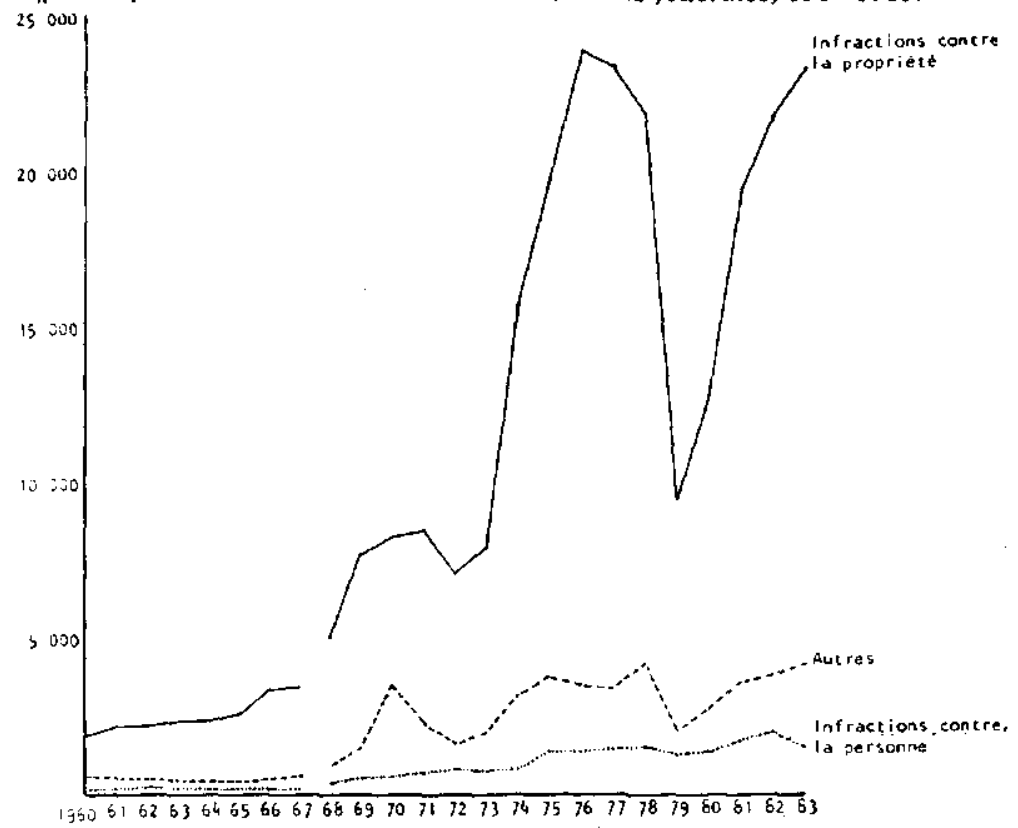

Source : Statistique Canada, Jeunes délinquants, annuel. Les infractions contre la propriété groupent ce que l'on retrouve dans le tableau 1 sous les rubriques de vols, introductions par effraction et autres infractions contre la propriété.

tableau 2 et illustrées dans le graphique 2. Les tendances observées suggèrent que ces vingt-quatre années peuvent être groupées en trois périodes.

La première période s'étend de 1960 à 1973. Le nombre d'affaires rapportées y est beaucoup moins élevé que par la suite, se situant à 2618 en 1960 . On y note une stabilité relative jusqu'en 1965, suivie en 1966 d'une augmentation qui s'accentuera surtout à compter de 1969. La progression des diverses catégories d'infractions suit un rythme plus ou moins similaire. Les infractions contre la propriété (et tout particulièrement les vols) dominent par leur volume, les infractions contre la personne ${ }^{53}$ ne comptant que pour

53. Les vols qualifiés ont été inclus dans les infractions contre la personne. 


\section{FIGURE 2}

Mesures ordonnées par le tribunal de la leunesse (Québec) pour des infractions au code criminel et autres lois fédérales, 1960-1983

(Nombres d'ordonnances).

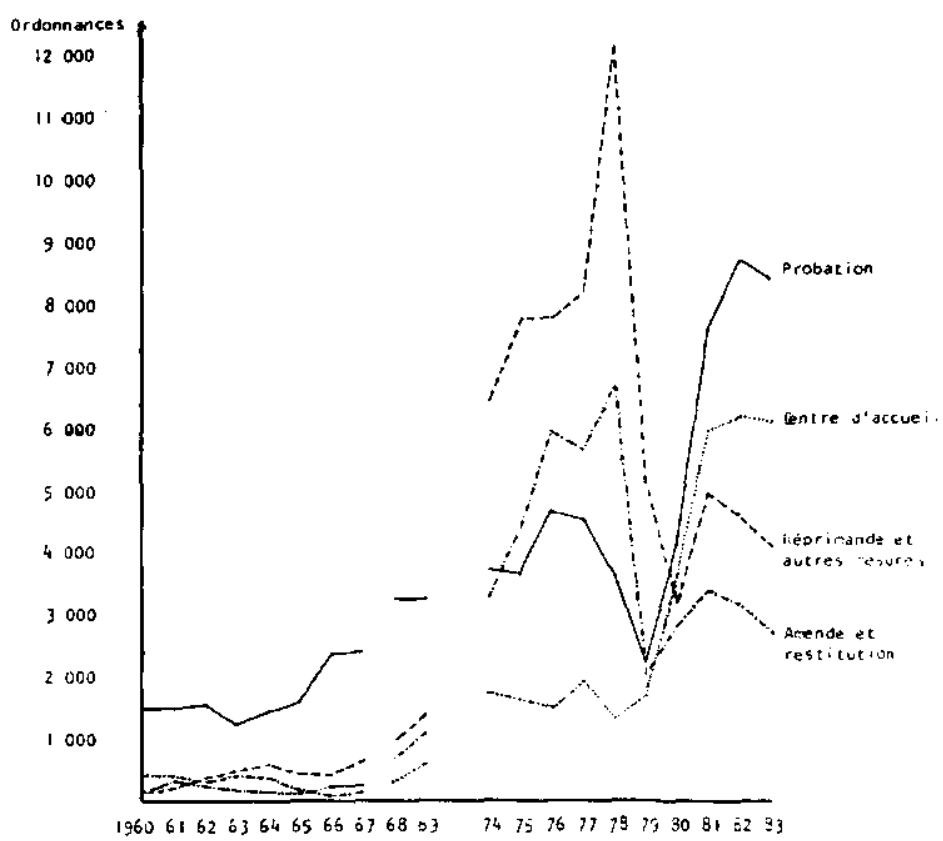

Source: Statistique Canada, Jeunes délinquants, annuel.

un maigre $5,9 \%$ du total. Pour les années concernant lesquelles l'information est disponible (1960-1969), la probation est nettement la plus utilisée des mesures $(61,5 \%)$, suivie des mesures ne comportant pas de surveillance $(30,4 \%)$ et, loin derrière, des placements en centre d'accueil $(8,1 \%)$.

La seconde période couvre les années 1974-1978. Elle est marquée par une explosion dans le nombre des affaires rapportées : dans la seule année 1974, ce nombre augmente de $33,6 \%$. Les infractions contre la personne n'y comptent toujours que pour $5,2 \%$ des affaires, alors que les infractions contre la propriété dominent toujours. Dans ce dernier groupe, une phénomène nouveau s'amorce : la préséance des introductions par effraction sur les vols commence en 1975 et s'accentuera à compter de 1977. Les mesures 
n'impliquant aucune surveillance (et tout particulièrement la réprimande) deviennent de loin les plus utilisées par les juges $(70,7 \%)$, la probation passant au second rang $(20,9 \%)$ et les placements en centre d'accueil demeurant encore loin derrière $(8,4 \%)$. L'accroissement soudain du nombre de causes et du volume des mesures n'impliquant qu'une intervention minimale de la part du tribunal suggère que les nouvelles affaires ainsi référées à la cour en étaient que les juges estimaient sans grande gravité. D'où la nature des décisions.

La troisième période va de 1979 à 1983 . C'est celle du mécanisme de déjudiciarisation régi par la Loi sur la protection de la jeunesse. On assiste tout d'abord à une chute saisissante du nombre des affaires en 1979 (passant de 27581 à 12 839), puis à une remontée qui s'amorce dès 1980 pour revenir rapidement aux niveaux de la période antérieure. La chute temporaire touche toutes les catégories d'infractions; elle est toutefois beaucoup moins évidente pour les infractions contre la personne que pour les autres catégories. Cette chute dans le nombre d'affaires se traduit par une baisse correspondante dans le recours aux diverses mesures en milieu ouvert, mais elle n'empêche pas une augmentation du nombre de placements en centre d'accueil de s'amorcer au même moment, dès 1979. Le retour au volume d'affaires antérieur donne lieu à un recours croissant à l'ensemble des mesures, avec un accent tout particulier sur la probation et les placements en centres d'accueil. Au total, donc, probation compte pour $34,0 \%$ des ordonnances rendues au cours de ces cinq années; les placements en centre d'accueil suivent avec $25,9 \%$ des affaires (soit un pourcentage trois fois plus élevé que celui que l'on avait connu antérieurement); la réprimande (et autres mesures) ainsi que l'amende (et la restitution) suivent avec respectivement $24,1 \%$ et $16 \%$ des mesures. Et, comme l'illustre clairement le graphique 2 , l'avance de la probation et des placements en centre d'accueil sur les mesures n'impliquant pas de surveillance de la part du tribunal se fait nettement plus claire à compter de 1981. Il est intéressant de noter que cette situation est exactement à l'inverse de celle de la période précédente (1974-1978), où les mesures sans surveillance avaient été - et de loin - les plus utilisées. Certaines de ces données ne trouvent pas d'explication aisée. Ainsi, comment expliquer que le nombre d'ordonnances de placement en centre d'accueil ait augmenté de près de $450 \%$ entre 1978 et 1983, alors que le nombre de places ne semble pas avoir augmenté au cours 
de la même période? Certaines hypothèses explicatives auraient besoin d'être vérifiées. Quoi qu'il en soit, il semble se dégager de ces données que les situations concernant lesquelles des mesures sont ordonnées et sont perçues par les juges comme requérant des interventions plus considérables qu'antérieurement. Les situations sont-elles effectivement plus sérieuses? Ou est-ce la perception que les intervenants en ont qui a changé? Ou encore les pratiques ont-elles évolué vers un interventionnisme plus poussé? Voilà autant d'interrogations que ces chiffres soulèvent sans malheureusement $\mathrm{y}$ apporter de réponses.

Une autre question est posée par ces données: celle de l'extension du contrôle social officiel par la mise sur pied en 1979 d'un mécanisme formel de déjudiciarisation. Un des objectifs généralement assignés à de tels mécanismes réside dans la réduction du contrôle social officiel : on entend éviter la référence au tribunal à des jeunes pour lesquels elle n'est pas vraiment nécessaire. Les données citées plus haut suggèrent que cette réduction du nombre de références au tribunal n'a été que très temporaire, ne s'étant vraiment fait sentir qu'en 1979 et 1980 . Or, ce phénomène doit être vu en conjonction avec un autre : l'évolution des signalements faits par les policiers. Alors que les statistiques policières ${ }^{54}$ faisaient état d'un nombre moyen de 25033 jeunes signalés aux tribunaux pour des infractions au Code criminel entre 1974 et 1978 , le nombre de jeunes signalés aux directeurs de la protection de la jeunesse monta respectivement à 31774,40668 et 41675 pour les années 1979, 1980 et 1981 . Une légère baisse à 38027 et 38295 pour 1982 et 1983 n'empêche pas la moyenne de s'établir à 38088 pour les années 1979-1983, soit une augmentation de $52 \%$ par rapport à la moyenne des cinq années antérieures.

Certains observateurs ont suggéré que ces chiffres reflétaient une importante augmentation dans la délinquance juvénile, et que la mise en vigueur de la nouvelle Loi sur la protection de la jeunesse en 1979 devait en être tenue responsable. Toutefois, une telle conclusion se fonde sur les postulats contestables que les variations dans les statistiques officielles ne peuvent être expliquées que par des variations dans le volume réel de la délinquance, et qu'une augmentation de délinquance coïncidant avec une réforme

54. Ces données sont tirées des publications annuelles pour 1981 et 1983 du ministère de la Justice du Québec, Statistiques; criminalité et application des règlements de la circulation au Québec. 
législative doit être attribué à cette réforme. Si la possibilité de tels effets ne peut être totalement exclue, il est bien connu que les statistiques officielles peuvent être influencées par d'autres facteurs.

L'on sait notamment qu'au Québec, en 1979, des policiers reçurent la directive de n'exercer aucune discrétion et de signaler aux directeurs de la protection de la jeunesse tous les cas pour lesquels la preuve de l'infraction était suffisante. Par ailleurs, des policiers sentirent peut-être moins le besoin de décider de ne prendre aucune action dans un certain nombre de cas, estimant que c'était devenu le rôle du directeur de la protection de la jeunesse de prendre une telle décision. En fait, l'introduction d'une étape intermédiaire entre la cour et les policiers venait modifier la nature des décisions que ceux-ci avaient à prendre. Antérieurement, les policiers choisissaient entre renvoyer le jeune chez lui et intenter des poursuites. Avec la nouvelle loi, ils devaient désormais choisir entre renvoyer le jeune chez lui et le signaler à une autre personne qui, elle, déciderait si des poursuites devaient être initiées. Il est fort possible que, dans des cas où antérieurement ils auraient décidé après hésitation de n'intenter aucune poursuite, des policiers aient tout simplement envoyé l'affaire au directeur de la protection de la jeunesse en lui laissant le soin de prendre une décision. Comme l'a souligné $\mathrm{Doob}^{55}$, la formalisation de la déjudiciarisation a transformé la décision du policier en une "non-décision», lui retirant une large part de ce qu'elle pouvait avoir de sérieux aux yeux du policier. La conséquence semble être que les policiers décident moins fréquemment de ne prendre aucune mesure et signalent plus facilement les cas. Des jeunes font donc l'objet d'une référence au directeur de la protection de la jeunesse alors que. sous l'ancien régime, ils auraient simplement été renvoyés chez eux. Paradoxalement, un régime qui visait à réduire le contrôle social officiel l'a peut-être élargi : contrairement aux objectifs visés, plus d'affaires sont signalées par les policiers et la réduction dans le nombre de causes se rendant au tribunal n'a été que très passagère. Ne s'agirait-il pas là d'un de ces phénomènes que Raymond Boudon

55. DOOB, Anthony N. (1983), a Turning decisions into non-decision», dans R. Corrado, M. Leblanc et J. Trépanier (édit.), Current Issues in Juvenile Justice, Toronto, Butterworths, p. 147-168. 
a nommés «effets pervers» ${ }^{56}$ ? Le changement a-t-il été à cet égard pour le mieux?

\section{EN GUISE DE CONCLUSION}

Les observations qui précèdent illustrent bien un certain nombre de changements qui ont transformé la justice des mineurs québécoise au cours des vingt-cinq dernières années. Les grands changements législatifs ont mis un accent nouveau sur les droits des jeunes et sur la déjudiciarisation; l'esprit de la loi a été considérablement modifié, particulièrement en ce qui a trait aux mineurs délinquants. Le recours au tribunal se fait aujourd'hui beaucoup plus fréquent qu'il y a quelques années, et les politiques de déjudiciarisation semblent avoir été sans succès pour contrer ce phénomène. Et là où le tribunal ordonne des interventions, on semble aller plus loin qu'auparavant. Si les statistiques officielles rendent compte d'une évolution réelle, il semble donc qu'on intervienne plus qu'autrefois. D'autres aspects fort importants de la transformation de la justice des mineurs auraient pu être abordés si l'espace l'avait permis. Que l'on pense par exemple à l'impact de la professionnalisation et de l'augmentation du personnel, des modifications apportées à la nomination des juges, de la création de l'aide juridique, de la bureaucratisation des services, de l'évolution des relations de travail, de la réduction des "caseloads", de l'intégration des agents de probation aux centres de services sociaux, de la transformation en profondeur de ressources telles que les centres d'accueil et les services sociaux, et ainsi de suite. La seule évocation de ces points suffit à rappeler que, même bien au-delà des aspects soulevés dans cet article, la justice des mineurs a un visage fort différent de celui qu'elle présentait en 1960.

56. BOUDON, Raymond (1977), Effets pervers et ordre social, Paris, Presses universitaires de France. 
TABLEAU 1

Nombre d'alfaires référées au Tribunal de la jeunesse (Québec)

pour des infractions au Code criminel et autres lois tédérales, $1960-1983$

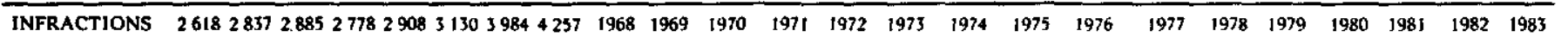

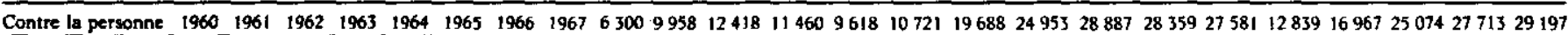
\begin{tabular}{lllllllllllllllllllllllllllllll}
\hline Vols & 108 & 128 & 199 & 121 & 155 & 129 & 130 & 146 & 315 & 623 & 681 & 749 & 833 & 773 & 889 & 1383 & 1403 & 1470 & 1571 & 1254 & 1353 & 1853 & 2006 & 1640
\end{tabular} Introductions par effraction

$\begin{array}{lllllllllllllllll}1211 & 317132912891454159819091939 & 27094094 & 4515 & 45544128 & 4039 & 6987 & 7732 & 9484 & 7820 & 7768 & 3105 \quad 3285 & 4765 & 5443 \quad 6081\end{array}$

Autres infractions

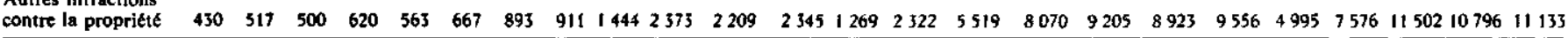
\begin{tabular}{llllllllllllllllllllllllllll}
\hline Autres & 274 & 336 & 339 & 418 & 391 & 395 & 602 & 684 & 864 & 1292 & 1510 & 1542 & 1656 & 1568 & 3202 & 3944 & 5220 & 6736 & 4518 & 1429 & 2003 & 3274 & 5620 & 6140
\end{tabular} $\begin{array}{llllllllllllllllllllllllllllll}\text { TOTAL } & 595 & 539 & 518 & 330 & 345 & 341 & 450 & 577 & 968 & 1576 & 3503 & 2270 & 1732 & 2019 & 3091 & 3824 & 3575 & 3410 & 4168 & 2056 & 2750 & 3680 & 3848 & 4203\end{array}$

Source : Statistique Canada, leunes dëlinquants, annuel.

\section{TABLEAU 2}

Nombre d'ordonnances rendues par le tribunal de la ieunesse (Ouébec) pour des infractions au Code criminel er autres lois fédérales. 1960-1983

\begin{tabular}{|c|c|c|c|c|c|c|c|c|c|c|c|c|c|c|c|c|c|c|c|c|}
\hline MESURES & 1960 & 1961 & 1962 & 1963 & 1964 & 1965 & 1966 & 1967 & 1968 & 1969 & 1974 & 1975 & 1976 & 1977 & 1978 & 1979 & 1980 & 1981 & 1982 & 1983 \\
\hline Centre d'accueil & 181 & 306 & 260 & 198 & 186 & 152 & 245 & 266 & 292 & 575 & 1745 & 1631 & 1495 & 1896 & 1369 & $172 j$ & 3598 & 5955 & 6200 & 6143 \\
\hline Probation & 1492 & 1502 & 1554 & 1255 & 1420 & 1635 & 2318 & 2402 & 3255 & 3265 & 3746 & 3636 & 4619 & 4506 & 3607 & 2242 & 4109 & 7523 & 8696 & 8570 \\
\hline Amende, restitution & 428 & 383 & 279 & 389 & 330 & 173 & 115 & 176 & 725 & 108 & 3289 & 4379 & 5917 & 5677 & 6635 & 2016 & 2796 & 3392 & 3646 & 2744 \\
\hline Réprimande et autres & 183 & 233 & 387 & 494 & 560 & 475 & 438 & 685 & 978 & 1592 & 6416 & 7736 & 7750 & 8158 & 12085 & 5089 & 3225 & 4950 & 4566 & 4158 \\
\hline
\end{tabular}

